Радиология — практика. 2021. № 5.

Radioilogy — practice. 2021. No. 5.

\title{
ПРОДОЛЖЕННОЕ МЕДИЦИНСКОЕ ОБРАЗОВАНИЕ
}

Обзор литературы.

https://doi.org/10.52560/2713-0118-2021-5-115-128

\section{Лучевая визуализация бронхоэктазов у детей и подростков (обзор литературы с собственными наблюдениями)}

\author{
П. В. Гаврилов \\ ФГБУ «Санкт-Петербургскии̃ научно-исслеgовательскии̃ институт \\ фртизиопульмонологиu» Минзgрава России, Санкт-Петербург
}

\section{Реферат}

Своевременное выявление бронхоэктазов у детей имеет важное прогностическое значение. Важность современного выявления бронхоэктазов у детей обусловлена возможной их обратимостью со временем при эффективном лечении, а также рядом существенных различий между бронхоэктазами с дебютом в детском возрасте и дебютом у взрослых, которые могут отражать разные фенотипы бронхоэктазов. У взрослых пациентов с бронхоэктазами, развившимися в детстве, течение патологического процесса более тяжелое и более худший прогноз по сравнению с бронхоэктазами, начавшимися во взрослом возрасте. Европейское респираторное общество в 2020 году предложило новую формулировку термина «бронхоэктаз» у детей и подростков, и в настоящее время под данным термином подразумевается аномальное расширение бронхов при компьютерной томографии (КТ) грудной клетки, которое при раннем обнаружении может быть обратимым. КТ является в настоящее время «золотым стандартом» для подтверждения бронхоэктазов у детей и подростков. Данный метод входит в минимальный набор тестов для детей и подростков с подозрением на бронхоэктазы согласно рекомендациям Европейского респираторного общества. При проведении КТ рекомендовано выполнение непрерывного спирального сканирования с коллимацией 1 мм, исследование проводится без внутривенного контрастирования с возможностью применения низкодозовых протоколов. Для оценки состояния бронхов у детей и подростков необходимо использовать педиатрические критерии

\footnotetext{
* Гаврилов Павел Владимирович, кандидат медицинских наук, ведущий научный сотрудник, руководитель направления «Лучевая диагностика» Федерального государственного бюджетного учреждения «СанктПетербургский научно-исследовательский институт фтизиопульмонологии» Министерства здравоохранения Российской Федерации.

Адрес: 191036, г. Санкт-Петербург, Лиговский пр., д. 2-4.

Телефон: +7 (812) 775-75-55. Электронная почта: spbniifrentgen@mail.ru

ORCID.org/0000-0003-3251-4084
}

Gavrilov Pavel Vladimirovich, Ph. D. Med., leading Researcher, Head of the Department of Radiology SaintPetersburg State Research Institute of Phthisiopulmonology.

Address: 2-4, Ligovsky pr., St. Petersburg, 191036, Russia.

Phone number: +7 (812) 775-75-55. E-mail: spbniifrentgen@mail.ru

ORCID.org/0000-0003-3251-4084

(C) П. В. Гаврилов. 
бронхо-артериального соотношения $(>0,8)$. При этом лучевые методы не являются определяющими в установлении причин бронхоэктазов и только в редких случаях позволяют помочь в установлении причин бронхоэктазов. Динамическое наблюдение за бронхоэктазами у детей и подростков определяется индивидуально. Следует рассмотреть возможность повторной компьютерной томографии грудной клетки, чтобы ответить на вопрос, который изменит лечение.

Ключевые слова: компьютерная томография органов грудной полости, бронхо-артериальное соотношение, симптом перстня, симптом трамвайных рельсов.

Literature review.

\title{
Radiology Imaging of Bronchiectasis in Children and Adolescents (Literature Review with Own Observations)
}

\author{
P. V. Gavrilov
}

Saint Petersburg Research Institute of Phthisiopulmonology, Ministry of Healthcare of Russia, Saint Petersburg, Russia

\section{Abstract}

Timely detection of bronchiectasis in children is of great prognostic value. The importance of modern bronchiectasis in children is due to their possible reversibility over time with effective treatment, as well as a number of significant differences between bronchiectasis in childhood and onset in children, which may reflect different phenotypes of bronchiectasis. In adult patients with bronchiectasis that began in childhood, the pathological process has a more severe and worse prognosis compared to bronchiectasis that began in adulthood. In 2020, the European Respiratory Society proposed a new formulation of the terminal «bronchiectasis» for children and adolescents, now this term refers to an abnormal expansion of the bronchi by computed tomography (CT) of the chest, which, if detected early, can be reversible. CT is the true «gold standard» for confirming bronchiectasis in children and adolescents. This method is included in the minimum set of tests for children and adolescents with suspected bronchiectasis according to the recommendations of the European Respiratory Society. When conducting CT, it is recommended to perform a continuous spiral scan with a collimation of $1 \mathrm{~mm}$, the study is carried out without intravenous use using low-dose protocols. To assess the state of the bronchi in children and adolescents, it is necessary to use the pediatric norms of the broncho-arterial ratio $(>0.8)$. At the same time, radiation methods are not decisive in establishing the causes of bronchiectasis and only in rare cases can help in establishing bronchiectasis. Dynamic observation of bronchiectasis for children and adolescents is determined individually. Consideration should be given to repeat chest computed tomography scans to answer the question that will change treatment.

Key words: Chest Computed Tomography, Broncho-Arterial Ratio, Tram-Track Sign, Signet Ring Sign.

\section{Актуальность}

Под классическим определением бронхоэктазов, согласно глоссарию терминов для торакальной визуализации, составленному Fleischner Society, понимается необратимая локализованная или диффузная дилатация бронхов, обычно в результате хронической инфекции, проксимальной обструкции дыхательных 
путей или врожденной бронхиальной патологии [13]. Аналогичное по смыслу определение, в котором под термином «бронхоэктаз» подразумевается локализованное необратимое расширение бронха, сопровождающееся воспалительными изменениями в стенке и окружающей паренхиме и развитием фиброза, дается в клинических рекомендациях по бронхоэктазам у детей, подготовленных Союзом педиатров России [1, 2].

Таким образом, одним из основных критериев для термина «бронхоэктаз» у детей и подростков являлось наличие необратимости изменений с необходимостью дифференциальной диагностики с обратимыми расширениями бронхов при острых инфекционных процессах в легких (так называемой функциональной бронходилатацией). Для этого требуется выполнение как минимум двух компьютерных томографий (КТ), что связано с повышением радиационного воздействия на ребенка.

Учитывая данный фактор, а также то, что при проведении исследований по динамическому наблюдению за бронхоэктазами у детей расширение бронхов полностью исчезало или уменьшалось у части пациентов со средним интервалом в 21 мес, Европейское респираторное общество в 2020 году предложило изменить формулировку термина «бронхоэктаз» у детей и подростков, и в настоящее время под данным термином подразумевается аномальное расширение бронхов при КТ грудной клетки, которое при раннем обнаружении может быть обратимым [7, 11].

Точная распространенность бронхоэктазов в детской популяции достоверно неизвестна и, по данным различных источников, колеблется от 1,5 до 67 на 100 тыс. населения в зависимости от страны и этнической принадлежности. При этом данных об эпидемиологических исследованиях по распространенности бронхоэктазов у детей в Российской Федерации в литературе не найдено [2, 10, 16].

Важность современного выявления бронхоэктазов у детей обусловлена не только обратимостью со временем при эффективном лечении, но и тем, что существует ряд существенных различий между бронхоэктазами с дебютом в детском возрасте и дебютом у взрослых, которые могут отражать разные фенотипы бронхоэктазов. У взрослых пациентов с бронхоэктазами, развившимися в детстве, течение патологического процесса более тяжелое и более худший прогноз по сравнению с бронхоэктазами, начавшимися во взрослом возрасте [2, 14].

Таким образом, своевременное выявление бронхоэктазов у детей имеет важное прогностическое значение.

Цель: проиллюстрировать особенности визуализации бронхоэктазов у детей в контексте рекомендаций Европейского респираторного общества.

\section{Методы визуализации бронхоэктазов}

Для визуализации бронхоэктазов могут применяться такие методы лучевой диагностики, как классическая рентгенография легких в передней и боковой проекциях, КТ органов грудной полости и магнитно-резонансная томография органов грудной полости (МРТ).

Однако, согласно данным литературы, рентгенография является достаточно низкоинформативным методом для визуализации бронхоэктазов и преимущественно позволяет увидеть лишь косвенные признаки в виде усиления и деформации легочного рисунка. Необходимость применения данного метода 
в российских клинических рекомендациях оценивается как слабая рекомендация [1, 2]. Европейские клинические рекомендации не рассматривают рентгенографию как метод диагностики бронхоэктазов [7].

Учитывая, что, согласно российским клиническим рекомендациям, в критерии качества оказания медицинской помощи входит обязательное выполнение КТ при постановке диагноза бронхоэктазы, в целях снижения лучевой нагрузки целесообразно исключить рентгенографию легких из методов обследования пациентов с подозрением на бронхоэктазы. Исключением может служить лишь техническая невозможность сделать КТ.

В настоящее время появились публикации, посвященные возможностям MPT в диагностике и оценке бронхоэктазов в динамике [8, 12, 18]

К преимуществам МРТ относится то, что это метод визуализации без ионизирующего излучения и может отображать морфологические признаки заболеваний легких при более низком пространственном разрешении.

Но недостаточное в диагностическом плане качество изображения на сегодняшний день, долгое время исследования, необходимость проведения анестезиологического пособия у детей младшего возраста не позволяют широко применять данный метод. На сегодняшний день ни один мировой гайдлайн, посвященный бронхоэктазам, у пациентов без муковисцидоза не упоминает МРТ как метод визуализации. И на сегодняшний момент МРТ может быть рекомендована в основном для динамического наблюдения пациентов с муковисцидозом в специализированных центрах [15].
КТ является в настоящее время «золотым стандартом» для подтверждения бронхоэктазов у детей и подростков [1, 2].

Данный метод входит в минимальный набор тестов для детей и подростков с подозрением на бронхоэктазы согласно рекомендациям Европейского респираторного общества. При проведении КТ рекомендовано выполнение непрерывного спирального сканирования с коллимацией 1 мм, исследование проводится без внутривенного контрастирования с возможностью применения низкодозовых протоколов [6, 7].

Показанием к проведению КТ для подтверждения или исключения бронхоэктазов, по данным европейских клинических рекомендаций, является: рецидивирующий (> 3 эпизодов в год) длительный бактериальный бронхит и влажный/продуктивный кашель, не поддающийся лечению в течение четырех недель перорального приема антибиотиков $[6,7]$.

Российские клинические рекомендации указывают, что заподозрить бронхоэктазы и назначить КТ необходимо в следующих случаях:

- хронический кашель (продуктивный или без мокроты) на протяжении более чем 8 недель;

- персистирующие хрипы в легких, которые невозможно объяснить другими причинами;

- неполное разрешение пневмонии после адекватной терапии или повторные пневмонии одной и той же локализации;

- «астма», торпидная к адекватно назначенной и проводимой терапии;

- наличие респираторных симптомов у детей со структурными и/ или функциональными нарушени- 
ями желудочно-кишечного тракта и верхних дыхательных путей;

- кровохарканье [1].

\section{Комьютерно-томографические признаки бронхоэктазов у детей}

При КТ выделяют прямые признаки бронхоэктазов, к которым относятся: расширение диаметра просвета бронхов (увеличение бронхо-артериального соотношения), визуализация бронхов в пределах 1 см от поверхности плевры и отсутствие сужения просвета $>2$ см от бифуркации бронха. Также выделяют косвенные признаки в виде утолщения стенки бронхов, визуализации содержимого (слизи) в просвете бронхов и неравномерную пневматизацию легочной ткани в зоне расположения измененных бронхов $[1,2,5]$.

Также выделяют два так называемых симптома-«образа», характерных для бронхоэктазов: симптом трамвайных рельсов (Tram-track sign) и симптом перстня (Signet Ring Sign) [19].

Наиболее важным КТ-симптомом для диагностики бронхоэктазов является бронхо-артериальное соотношение (Broncho-arterial ratio). Данное соотношение определяется как соотношение диаметра внутреннего просвета бронха к наружном диаметру сопровождающей его артерии (рис. 1) и обычно измеряется на уровне от сегментарной до субсегментарной артерии [5, 7].

У детей и подростков среднее значение бронхо-артериального соотношения - 0,6-0,7, а расширением бронха считается увеличение значения бронхо-артериального отношения более 0,8 (у взрослых увеличение бронхо-артериального соотношения $>1(>1,5)$ является признаком бронхоэктазии) [6, 7].

Выраженное увеличение бронхо-артериального соотношения приводит к тому, что комбинация из кольцевидной структуры, которая является расширенным бронхом в поперечном сечении и меньшего диаметра прилегающей легочной артерии, напоминает перстень - симптом перстня (Signet Ring Sign) (рис. 2).

Следующим важным признаком бронхоэктаза является отсутствие сужения просвета бронха $>2$ см от бифуркации бронха. Данный признак в сочетании с утолщением стенок бронхов представляет собой комбинацию, напоминающую трамвайные пути (рис. 3) так называемый симптом трамвайных рельсов (Tram-track sign).

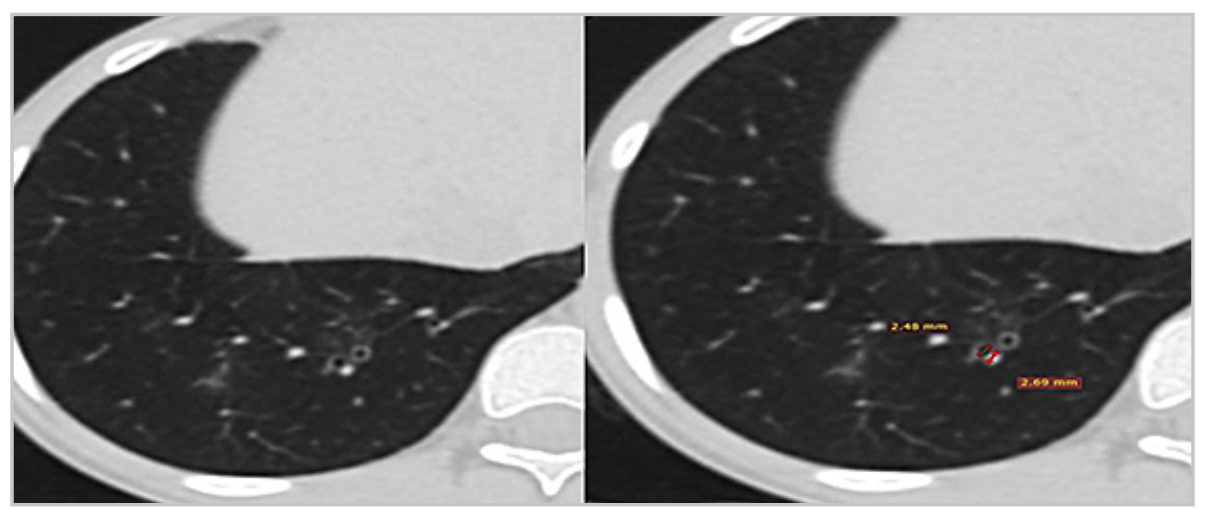

Рис. 1. Фрагменты компьютерных томограмм (аксиальная плоскость, легочное окно) ребенка 16 лет. Отношение диаметра внутреннего просвета бронха к наружном диаметру сопровождающей его артерии - 2,48/2,69 = 0,92 (расширение бронха) 


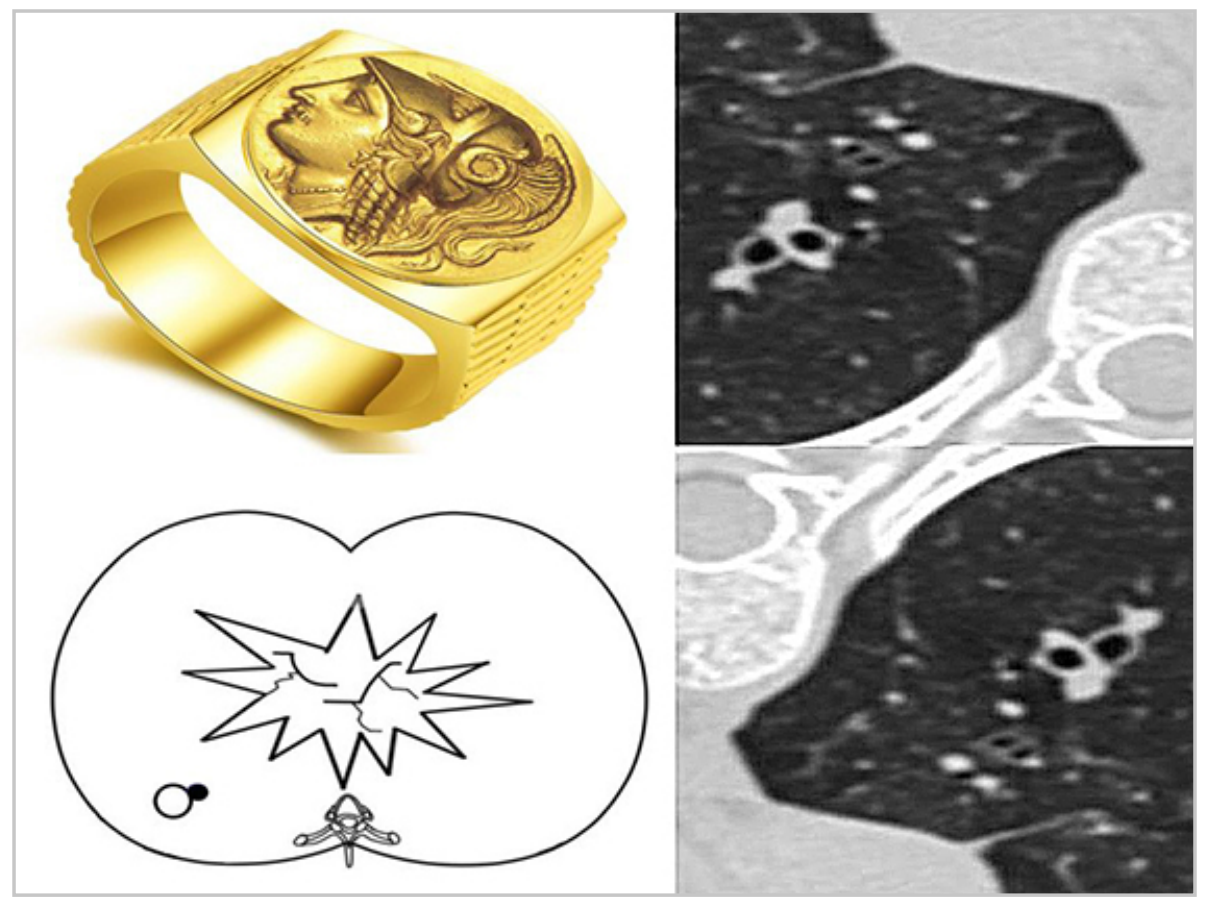

Рис. 2. Изображения, схема и фрагменты компьютерных томограмм в аксиальной плоскости, демонстрирующие расширенный бронх в поперечном сечении и меньшего диаметра прилегающую легочную артерию (симптом перстня)
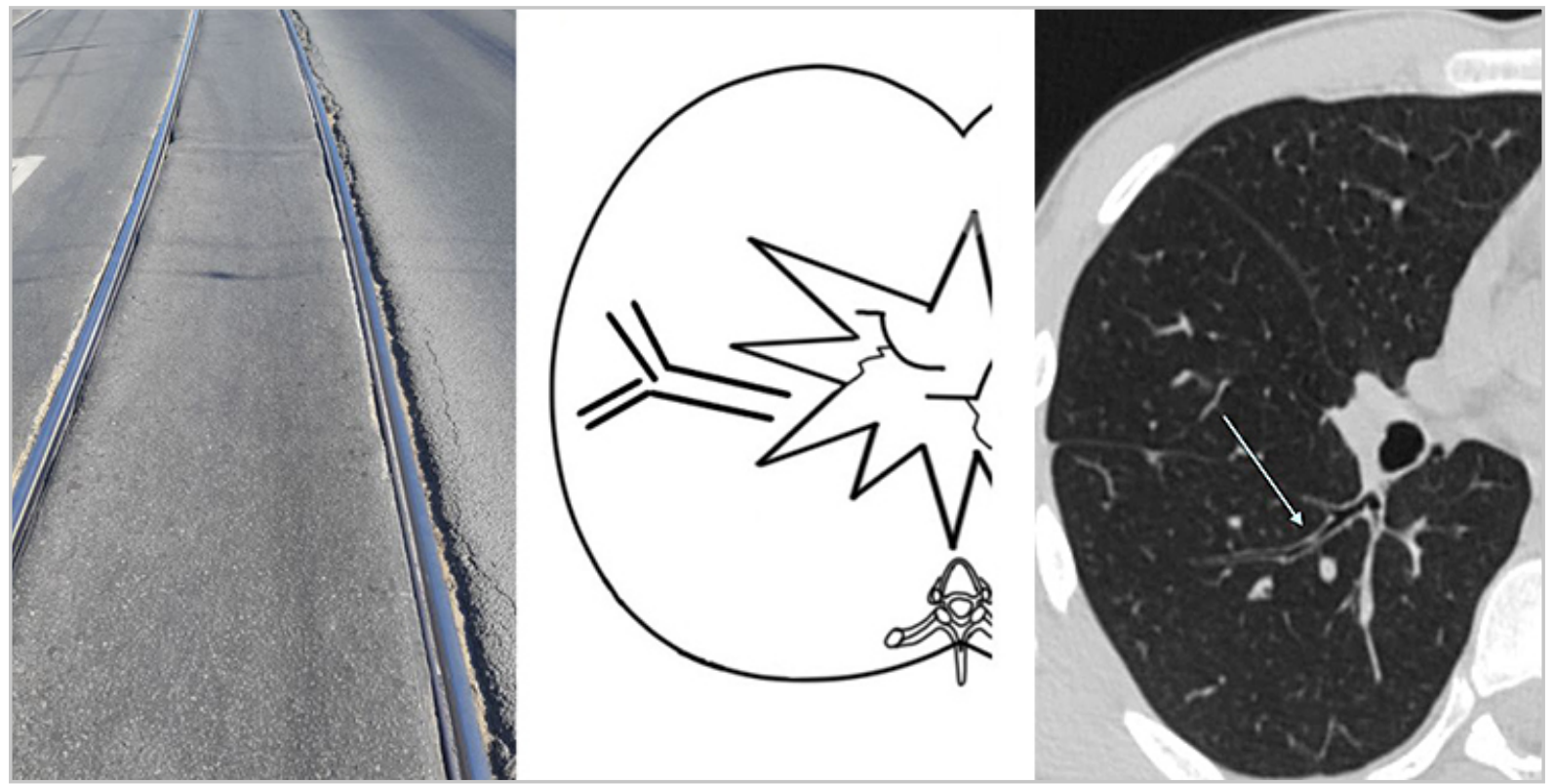

Рис. 3. Изображения, схема и фрагмент компьютерных томограмм в аксиальной плоскости, демонстрирующие симптом трамвайных рельсов (утолщенные стенки бронхов, окружающие воздухсодержащий просвет) 
Утолщение стенки бронха - это частый, но не постоянный признак бронхоэктазов. В норме толщина стенки составляет 20-30 \% от общего диаметра бронха (рис. 4). Следует отметить, что небольшое утолщение стенки может наблюдаться у здоровых людей, пациентов с бронхиальной астмой и курильщиков [16, 17, 18].

В нашей практике изолированное утолщение стенок бронхов без нарушения бронхо-артериального соотношения у детей и подростков не встречалось.

По скиалогической картине бронхоэктазы разделяют на цилиндрические, варикозные (веретенообразные) и мешотчатые (кистовидные бронхоэктазы) (рис. 5, $a-8$ ).

При цилиндрическом типе бронхоэктазов бронх равномерно расширен на всем протяжении. Такая форма бронхоэктазов не способствует скоплению большого количества секрета.

При варикозном (веретенообразном) характере расширения бронха стенка бронха расширяется неравномер- но с множественными мелкими выпячиваниями.

При мешотчатом (кистозном) характере бронхоэктазов отмечается выраженное расширение просвета бронха, напоминающее шаровидное или овальное полостное образование. Данные бронхоэктазы часто тяжело дифференцировать с полостями (рис. 6), что требует использования многоплоскостных реконструкций и проекции минимальной интенсивности (MinIP). В таких бронхоэктазах часто скапливается секрет и гной с формированием уровней жидкости.

По мере прогрессирования процесса цилиндрические бронхоэктазы на фоне хронических рецидивирующих воспалительных процессов и развития перибронхиального склероза трансформируются сперва в варикозные, а потом в мешотчатые.

Особняком стоят так называемые ателектатические бронхоэктазы, возникающие в зоне частичного или полного ателектаза. Часть авторов выделяют их

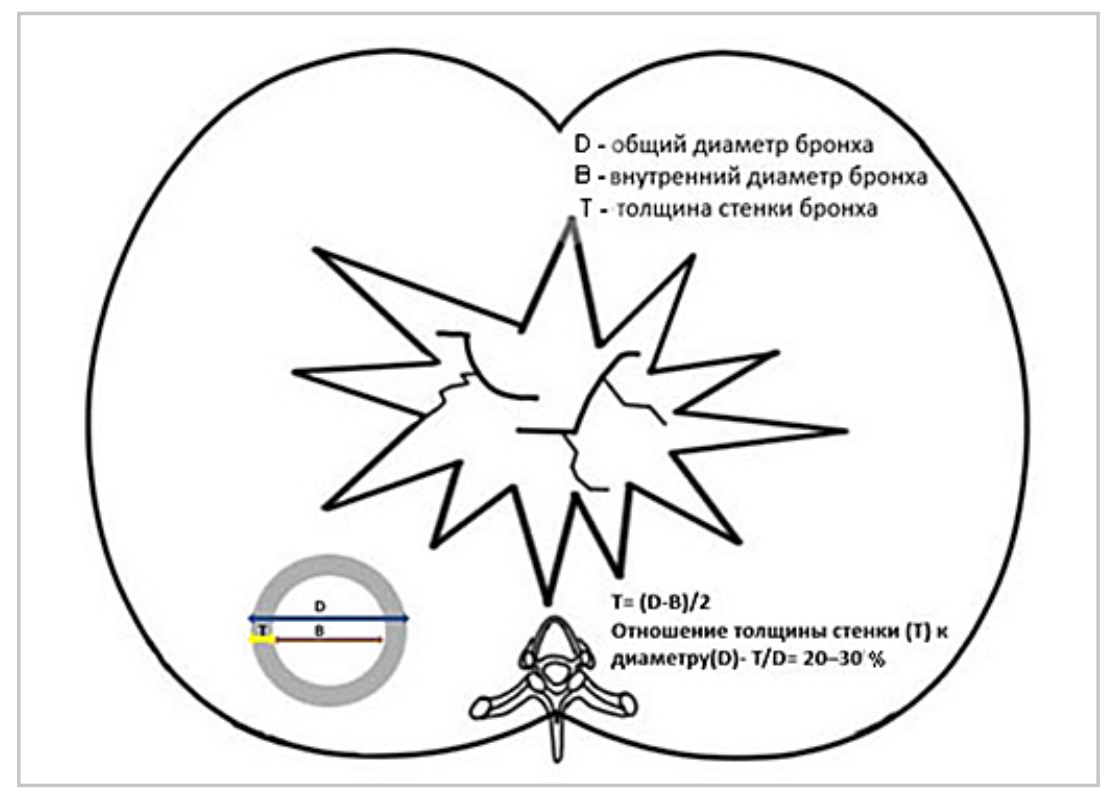

Рис. 4. Схема оценки толщины стенки бронха 


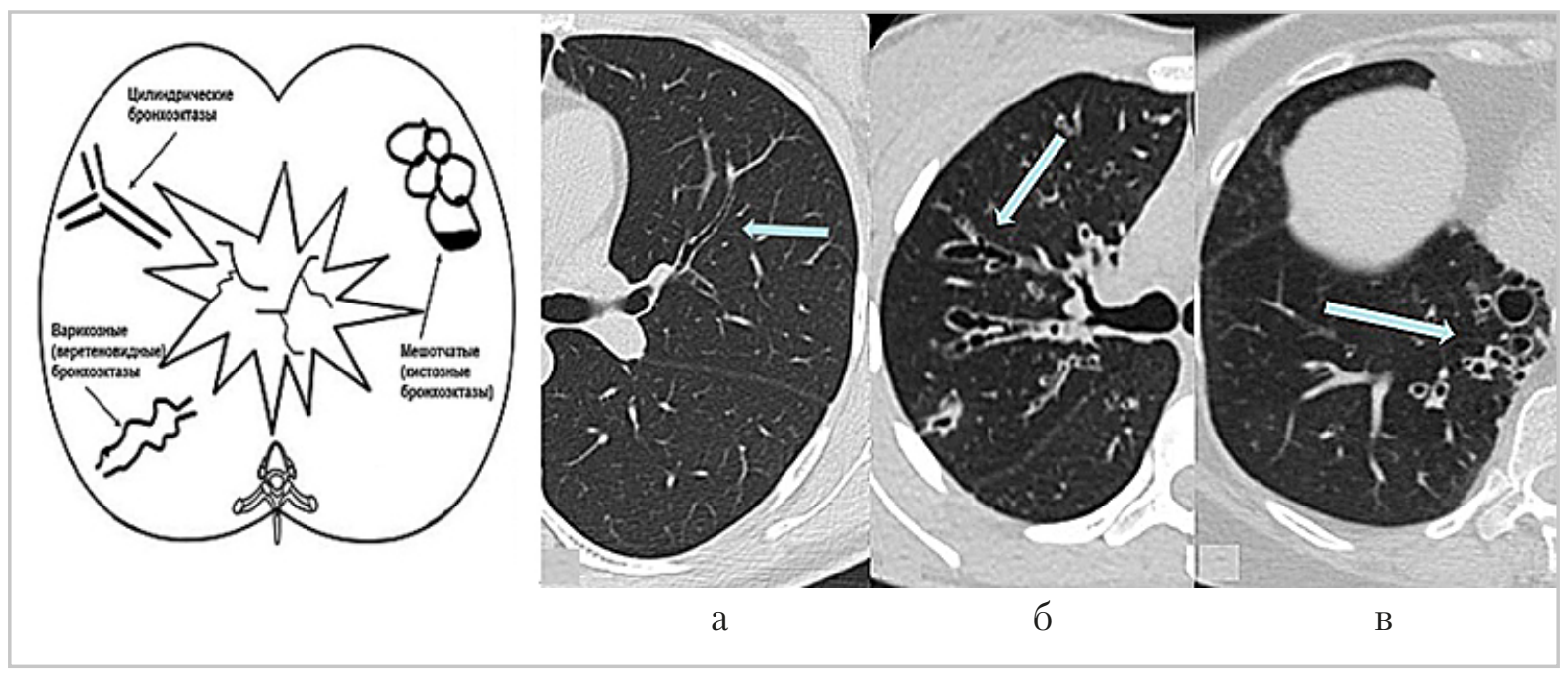

Рис. 5. Схема и фрагменты компьютерных томограмм в аксиальной плоскости, демонстрирующие различные типы бронхоэктазов: $a$ - цилиндрические бронхоэктазы; $\sigma$ - варикозные (веретеновидные) бронхоэктазы; в - мешотчатые (кистозные бронхоэктазы)

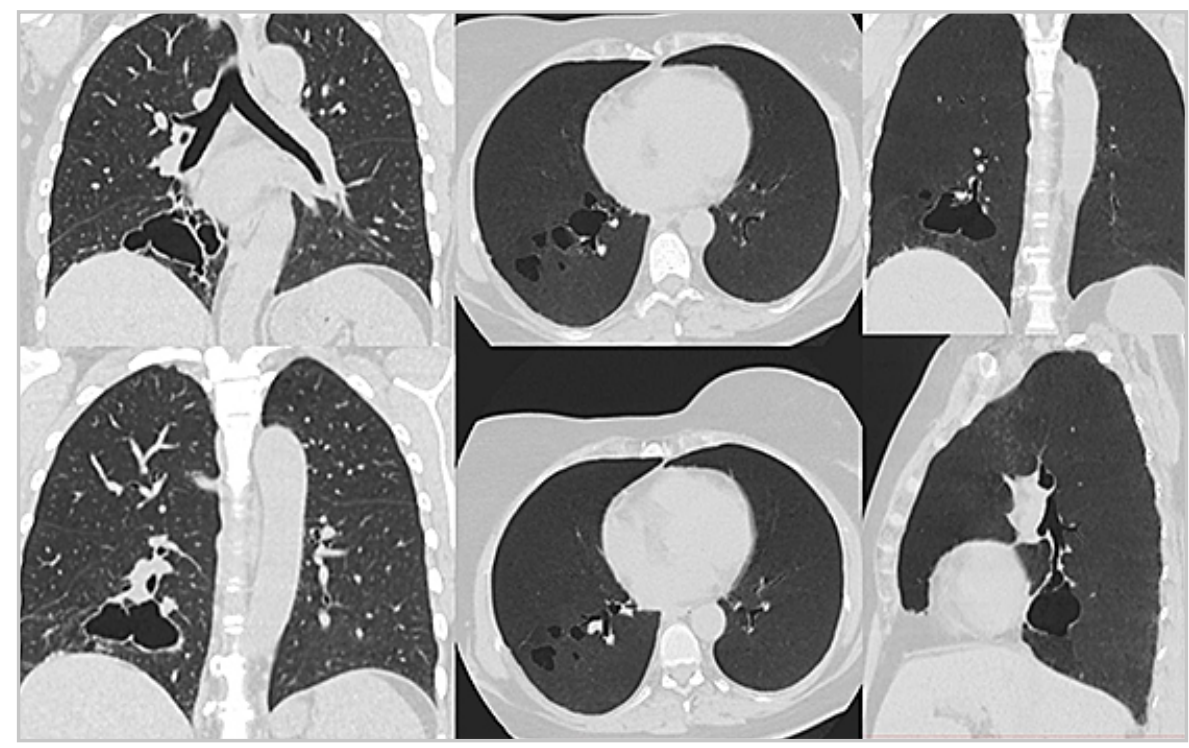

Рис. 6. Компьютерные томограммы в «легочном» окне и с применением проекции минимальной интенсивности, демонстрирующие мешотчатые бронхоэктазы нижней доли правого легкого, симулирующие полость. Применение реконструкций позволяет четко высказаться о характере патологии

как отдельный вид бронхоэктазов [2, 3], но при этом в скиалогической картине ателектазов необструктивного характера присутствует так называемый симптом воздушной бронхографии, то есть само понятие необструктивного ателек- таза подразумевает визуализацию на его фоне просветов расширенных бронхов.

\section{Этиология бронхоэктазов у детей}

Клинически все бронхоэктазы можно разделить на две большие группы: брон- 
хоэктазы как проявление бронхоэктатической болезни и бронхоэктазы, являющиеся проявлением другой патологии (первичная цилиарная дискинезия, пороки развития бронхов и др.).

Следует отметить, что огромное количество этиологических факторов и состояний может являться причинами бронхоэктазов у детей (табл.).

Бронхоэктазы, вызванные различными патологическими состояниями, практически не отличаются друг от друга. Лучевые методы не являются определяющими в установлении причин бронхоэктазов и только в редких случаях (например, синдром Зиверта - Картагенера) позволяют помочь в установлении причин бронхоэктазов (рис. 7). Также по результатам КТ можно заподозрить некоторые причины бронхоэктазов, например, для нетуберкулезных микобактериозов харак-

\section{Причины развития бронхоэктазов у gетей}

\begin{tabular}{|c|c|}
\hline Группы патологических процессов & Нозологические формы \\
\hline \multirow{5}{*}{$\begin{array}{l}\text { Врожденные структурные } \\
\text { аномалии строения } \\
\text { бронхолегочной системы }\end{array}$} & синдром Вильямса - Кэмпбелла \\
\hline & синдром Мунье - Куна \\
\hline & трахеобронхомегалия \\
\hline & трахеомаляция \\
\hline & бронхомаляци \\
\hline \multirow{7}{*}{ Инфекционные процессы } & коклюш \\
\hline & корь \\
\hline & аденовирусная инфекция \\
\hline & бактериальные пневмонии \\
\hline & туберкулез \\
\hline & нетуберкулезный микобактериоз \\
\hline & аллергический бронхолегочный аспергиллез \\
\hline \multirow{9}{*}{$\begin{array}{l}\text { Первичные иммунодефицитные } \\
\text { состояния }\end{array}$} & агаммаглобулинемия \\
\hline & общий вариабельный иммунодефицит \\
\hline & селективная недостаточность иммуноглобулина А \\
\hline & $\begin{array}{l}\text { селективная недостаточность субклассов иммуно- } \\
\text { глобулина G }\end{array}$ \\
\hline & $\begin{array}{l}\text { тяжелый комбинированный иммунодефицит, атак- } \\
\text { сия-телеангиоэктазия (синдром Луи - Барр) }\end{array}$ \\
\hline & синдром Джоба (гипер-IgE-синдром) \\
\hline & хроническая гранулематозная болезнь \\
\hline & $\begin{array}{l}\text { дефицит транспортеров, связанных с презентацией } \\
\text { антигенов }\end{array}$ \\
\hline & дефекты комплемента \\
\hline \multirow{2}{*}{$\begin{array}{l}\text { Нарушения мукоцилиарного } \\
\text { клиренса }\end{array}$} & первичная цилиарная дискинезия (ПЦД) \\
\hline & муковисцидоз \\
\hline
\end{tabular}




\begin{tabular}{|c|c|}
\hline Группы патологических процессов & Нозологические формы \\
\hline \multirow{9}{*}{ Системные заболевания } & ревматоидный артрит \\
\hline & системная склеродермия \\
\hline & рецидивирующий полихондрит \\
\hline & синдром Мейенбурга - Альтхерра - Юлингера \\
\hline & анкилозирующий спондилит \\
\hline & синдромы Элерса - Данло \\
\hline & синдром Марфана \\
\hline & синдром Янга \\
\hline & метафизарная хондродисплазия, тип Мак-Кьюсика \\
\hline \multirow{3}{*}{ Обструкция бронха } & $\begin{array}{l}\text { обструкция бронха, вызванная внешними причина- } \\
\text { ми (лимфаденопатия, аномальный сосуд, опухоль) }\end{array}$ \\
\hline & $\begin{array}{l}\text { внутрибронхиальная обструкция инородным телом } \\
\text { вследствие аспирации }\end{array}$ \\
\hline & $\begin{array}{l}\text { внутрибронхиальная обструкция объемным образо- } \\
\text { ванием (опухоль, гранулема и т. д.) }\end{array}$ \\
\hline Идиопатические бронхоэктазы & причины не установлены \\
\hline
\end{tabular}

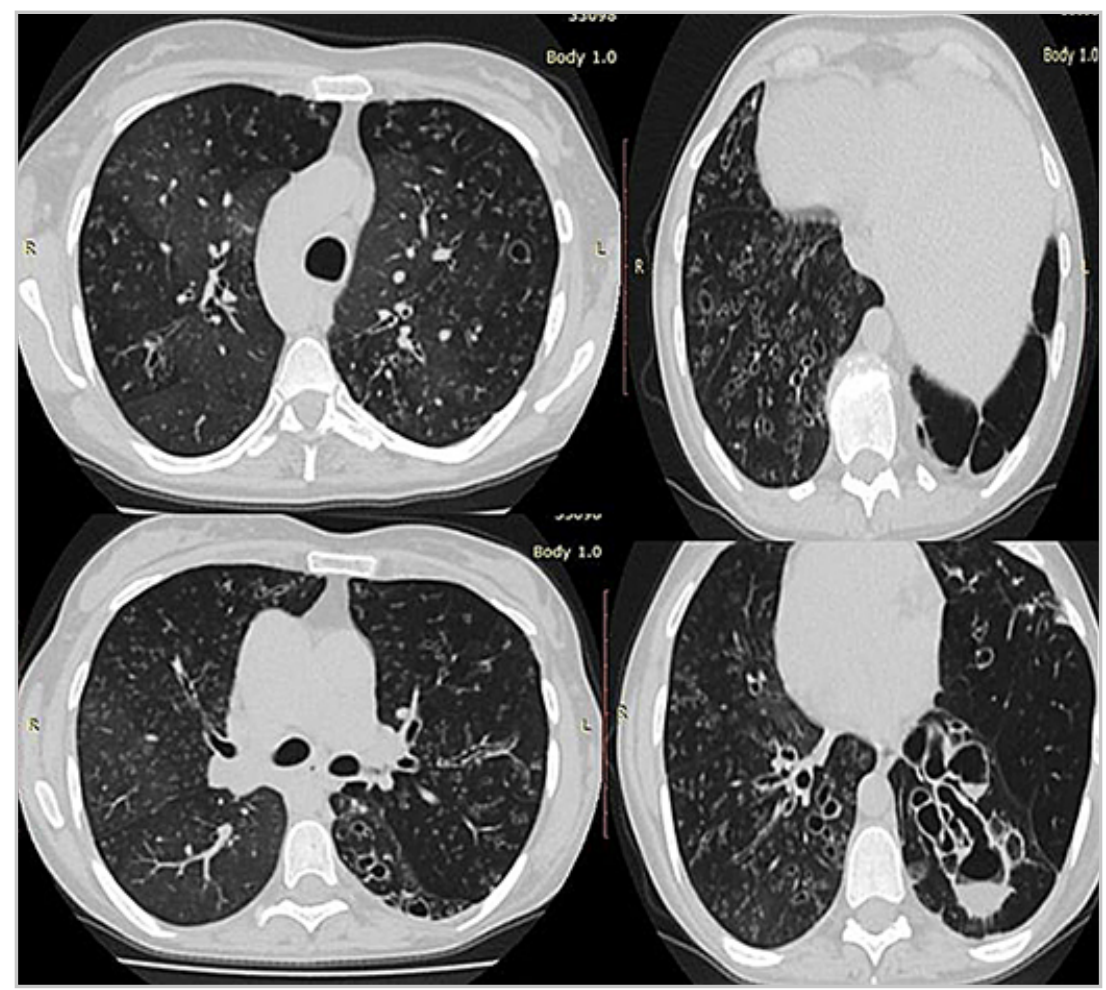

Рис. 7. Аксиальные срезы компьютерных томограмм в легочном окне пациента с синдромом Зиверта - Картагенера. Визуализируются транспозиция внутренних органов (situs viscerum inversus) и множественные мешотчатые бронхоэктазы вследствие аномалии мукоцилиарного клиренса 
терно поражение S 4,5 с обеих сторон, а для аллергического бронхолегочного аспергиллеза характерно наличие центральных бронхоэктазов [3, 4].

\section{Динамическое наблюдение бронхоэктазов у детей}

Не существует каких-либо сроков обязательного динамического наблюдения бронхоэктазов у детей и подростков. Решение о повторной компьютерной томографии грудной клетки принимается индивидуально в зависимости от клинического статуса и условий. Европейское респираторное общество рекомендует рассмотреть возможность повторной компьютерной томографии грудной клетки, чтобы ответить на вопрос, который изменит лечение [7].

\section{Заключение}

Бронхоэктазы у детей в настоящее время - актуальная проблема здравоохранения, поскольку у детей при раннем обнаружении могут быть обратимыми, а у взрослых пациентов с бронхоэктазами, развившимися в детстве, течение патологического процесса более тяжелое и более худший прогноз по сравнению с бронхоэктазами, начавшимися во взрослом возрасте.

КТ в настоящее время является «золотым стандартом» для подтверждения бронхоэктазов у детей и подростков.

Для оценки состояния бронхов у детей и подростков необходимо использовать педиатрические критерии бронхоартериального соотношения $(>0,8)$.

При этом лучевые методы не являются определяющими в установлении причин бронхоэктазов и только в редких случаях позволяют помочь в установлении причин бронхоэктазов.

Динамическое наблюдение за бронхоэктазами у детей и подростков определяется индивидуально. Следует рассмотреть возможность повторной компьютерной томографии грудной клетки, чтобы ответить на вопрос, который изменит лечение.

\section{Список литературы}

1. Баранов А. А., Намазова-Баранова Л. С., Симонова О. И., Вишнева Е. А., Селимзянова Л. Р., Середа Е. В., Розинова Н. Н., Цызина Е. Н., Катосова Л. К., Лазарева А. В., Горинова Ю. В., Кустова О. В. Клинические рекомендации «Бронхоэктазы у детей». М., 2016.

2. Баранов А. А., Намазова-Баранова Л. С., Симонова О. И., Вишнёва Е. А., Селимзянова Л. Р., Середа Е. В., Розинова Н. Н., Цыцина Е. Н., Катосова Л. К., Лазарева А. В., Горинова Ю. В., Кустова О. В. Бронхоэктазы у детей: обзор современных клинических рекомендаций // Педиатрическая фармакология. 2017. T. 14. № 1. С. 33-42. doi: 10.15690/ pf.v14i1.1699.

3. Зайщев А. А., Моисеев С. В. Бронхоэктазы, не обусловленные муковисцидозом: эпидемиология, диагностика и лечение // Клиническая фармакология и терапия. 2017. Т. 26. № 5. С. 19-24.

4. Степанов А. А., Бадалян А. Р., Мельникова A. О. Бронхоэктатическая болезнь у детей // Российский вестник перинатологии и педиатрии. 2018;63:(5):29-35. DOI: 10.21508/1027-4065-2018-63-5-29-35.

5. Лучевая анатомия. Грудь, живот, таз / под ред. Розадо-де-Кристенсон М. Л., Федерле М. П., Раман Ш. П. М.: Изд-во Панфилова, 2018. 1128 с.

6. Chang A. B., Bush A., Grimwood K. Bronchiectasis in children: diagnosis and treatment. Lancet. 2018 Sep 8;392(10150): 866-879. doi: 10.1016/S0140-6736(18) 31554-X. Erratum in: Lancet. 2018 Oct 6;392(10154):1196. 
7. Chang A. B., Fortescue R., Grimwood K., Alexopoulou E., Bell L., Boyd J., Bush A., Chalmers J. D., Hill A.T., Karadag B., Midulla F., McCallum G. B., Powell Z., Snijders D., Song W.J., Tonia T, Wilson C, Zacharasiewicz A., Kantar A. Task Force report: European Respiratory Society guidelines for the management of children and adolescents with bronchiectasis. Eur Respir J. 2021 Feb. 11:2002990. doi: 10.1183/13993003.02990-2020.

8. Chassagnon G., Brun A. L., Bennani S., Chergui N., Freche G., Revel M. P. Imagerie des dilatations des bronches [Bronchiectasis imaging]. Rev. Pneumol. Clin. 2018 Oct. 74(5):299-314. French. doi: 10.1016/j.pneumo.2018.09.009.

9. Diederich S., Jurriaans E., Flower C. D. Interobserver variation in the diagnosis of bronchiectasis on high-resolution computed tomography. Eur Radiol. 1996;6(6):801-6. doi: 10.1007/BF00240 675.

10. Floto R. A., Haworth C.S. Bronchiectasis. European Respiratory Society Monograph, V. 52. Sheffield, UK: European Respiratory Society; 2011. 261 p. doi: 10.1183/1025448x.erm5210.

11. Gaillard E. A., Carty H., Heaf D., Smyth $R$. L. Reversible bronchial dilatation in children: comparison of serial high-resolution computer tomography scans of the lungs. Eur. J. Radiol. 2003 Sep; 47(3):21520. doi: 10.1016/s0720-048x (02)001 22-5.

12. Goralski J. L., Stewart N. J., Woods J. C. Novel imaging techniques for cystic fibrosis lung disease. Pediatr Pulmonol. 2021 Feb; 56 Suppl 1 (Suppl 1): S40-S54. doi: 10.1002/ppul.24931.

13. Hansell D. M., Bankier A. A., MacMahon H., McLoud T. C., Müller N. L, Remy J. Fleischner Society: glossary of terms for thoracic imaging. Radiology. 2008
Mar; 246(3):697-722. doi: 10.1148/ radiol.2462070712.

14. King P. T., Holdsworth S. R., Farmer M., Freezer N., Villanueva E., Holmes $P$. W. Phenotypes of adult bronchiectasis: onset of productive cough in childhood and adulthood. COPD. 2009 Apr; 6(2):1306. doi: 10.1080/15412550902766934.

15. Murphy K. P., Maher M. M., O'Connor O.J. Imaging of Cystic Fibrosis and Pediatric Bronchiectasis. AJR Am. J. Roentgenol. 2016 Mar; 206(3):448-54. doi: 10.2214/ AJR.15.14437.

16. Ringshausen F. C., de Roux A., Diel R. et al. Bronchiectasis in Germany: a populationbased estimation of disease prevalence. Eur Respir J. 2015;46(6):1805-1807. doi: 10.1183/13993003. 00954-2015.

17. Remy-Jardin M., Remy J., Boulenguez C. et al. Morphologic effects of cigarette smoking on airways and pulmonary parenchyma in healthy adult volunteers: CT evaluation and correlation with pulmonary function tests. Radiology 1993; 186: 107-15.

18. Wielpütz M. O., Eichinger M., Biederer J., Wege S., Stahl M., Sommerburg O., Mall M. A., Kauczor H. U., Puderbach M. Imaging of Cystic Fibrosis Lung Disease and Clinical Interpretation. Rofo. 2016 Sep; 188(9):834-45. English. doi: 10.1055/s0042-104936.

19. Yudin A. L. Metaphorical signs in computed tomography of chest and abdomen. 2014. P. 1-176. DOI 10.1007/978-3-3194013-4 15.

\section{References}

1. Baranov A. A., Namazova-Baranova L. S., Simonova O. I., Vishneva E. A., Selimzyanova L. R., Sereda E. V., Rozinova N. N., Tzygina E. N., Katosova L. K., Lazareva A. V., Gorinova Yu. V., Kustova O. V. Clinical guidelines «Bronchiectasis in children» Moscow 2016 (in Russian). 
2. Baranov A. A., Namazova-Baranova L. S., Simonova O. I., Vishneva E. A., Selimzyanova L. R., Sereda E. V., Rozinova N. N., Tzygina E. N., Katosova L. K., Lazareva A. V., Gorinova Yu. V., Kustova $O$. $V$. Bronchiectases in Children: Overview of Current Clinical Guidelines. Pediatricheskaya farmakologiya - Pediatric pharmacology. 2017;14(1):33-42. doi: 10.15690/pf. v14i1. 1699 (in Russian).

3. Zaytsev A. A., Moiseev S. V. Non-cystic fibrosis bronchiectasis: epidemiology, diagnosis and current treatment. Clin. Pharmacol. Ther. 2017;26(5):19-24.

4. Stepanov A. A., Badalyan A. R., Melnikova A. O. Bronchoectatic Disease in Children. Ros Vestn Perinatol i Pediatr 2018; 63:(5):29-35 (in Russ). DOI: 10.215 08/1027-4065-2018-63-5-29-35 (in Russian).

5. Michael P. Federle Melissa L. Rosado-deChristenson Siva P. Raman //Imaging Anatomy: Chest, Abdomen, Pelvis, 2018. 1128 c. (in Russian).

6. Chang A. B., Bush A., Grimwood K. Bronchiectasis in children: diagnosis and treatment.Lancet.2018Sep..doi:10.1016/ S0140-6736(18)31554-X. Erratum in: Lancet. 2018 Oct. 6; 392(10154): 1196.

7. Chang A. B., Fortescue R., Grimwood K., Alexopoulou E., Bell L., Boyd J., Bush A., Chalmers J. D., Hill A. T., Karadag B., Midulla F., McCallum G. B., Powell Z., Snijders D., Song W.J., Tonia T., Wilson C., Zacharasiewicz A., Kantar A. Task Force report: European Respiratory Society guidelines for the management of children and adolescents with bronchiectasis. Eur Respir J. 2021. Feb. 11:2002990. doi: 10.1183/13993003.02990-2020.

8. Chassagnon G., Brun A. L, Bennani S., Chergui N., Freche G., Revel M. P. Imagerie des dilatations des bronches [Bronchiectasis imaging]. Rev. Pneumol.
Clin. 2018. Oct. 74(5):299-314. French. doi: 10.1016/j.pneumo.2018.09.009.

9. Diederich S., Jurriaans E., Flower $C$. $D$. Interobserver variation in the diagnosis of bronchiectasis on highresolution computed tomography. Eur Radiol. 1996;6(6):801-6. doi: 10.1007/ BF00240675

10. Floto R. A., Haworth C. S. Bronchiectasis. European Respiratory Society Monograph, Vol. 52. Sheffield, UK: European Respiratory Society; 2011.261 p. doi: $10.1183 /$ 1025448x.erm5210.

11. Gaillard E. A., Carty H., Heaf D., Smyth $R$. L. Reversible bronchial dilatation in children: comparison of serial highresolution computer tomography scans of the lungs. Eur. J. Radiol. 2003 Sep. 47(3):215-220. doi: 10.1016/s0720048x(02)00122-5.

12. Goralski J. L., Stewart N. J., Woods J. C. Novel imaging techniques for cystic fibrosis lung disease. Pediatr Pulmonol. 2021 Feb; 56 Suppl 1 (Suppl 1): S40-S54. doi: 10.1002/ppul.24931.

13. Hansell D. M., Bankier A. A., MacMahon H., McLoud T. C., Müller N. L., Remy J. Fleischner Society: glossary of terms for thoracic imaging. Radiology. 2008. Mar. 246(3):697-722. doi: 10.1148/ radiol.2462070712.

14. King P. T., Holdsworth S. R., Farmer M., Freezer N., Villanueva E., Holmes P. W. Phenotypes of adult bronchiectasis: onset of productive cough in childhood and adulthood. COPD. 2009. Apr. 6(2):1306. doi: 10.1080/15412550902766934.

15. Murphy K. P., Maher M. M., O'Connor O.J. Imaging of Cystic Fibrosis and Pediatric Bronchiectasis. AJR Am. J. Roentgenol. 2016. Mar; 206(3):448-54. doi: 10.2214/ AJR.15.14437

16. Ringshausen F. C., de Roux A., Diel R. et al. Bronchiectasis in Germany: a population- 
based estimation of disease prevalence. Eur. Respir. J. 2015;46(6):1805-1807. doi: 10.1183/13993003. 00954-2015.

17. Remy-Jardin M., Remy J., Boulenguez C. et al. Morphologic effects of cigarette smoking on airways and pulmonary parenchyma in healthy adult volunteers: CT evaluation and correlation with pulmonary function tests. Radiology. 1993; 186:107-15.
18. Wielpütz M. O., Eichinger M., Biederer J., Wege S., Stahl M., Sommerburg O., Mall M. A., Kauczor H. U., Puderbach M. Imaging of Cystic Fibrosis Lung Disease and Clinical Interpretation. Rofo. 2016 Sep; 188(9): 834-45. English. doi: 10.1055/s0042-104936.

19. Yudin A. L. Metaphorical signs in computed tomography of chest and abdomen// 2014. C. $1-176$.

\section{Сведения об авторе}

Гаврилов Павел Владимирович, кандидат медицинских наук, ведущий научный сотрудник, руководитель направления «Лучевая диагностика» Федерального государственного бюджетного учреждения «СанктПетербургский научно-исследовательский институт фтизиопульмонологии» Министерства здравоохранения Российской Федерации.

Адрес: 191036, г. Санкт-Петербург, Лиговский пр., д. 2-4.

Телефон: +7 (812) 775-75-55. Электронная почта: spbniifrentgen@mail.ru

ORCID.org/0000-0003-3251-4084

Gavrilov Pavel Vladimirovich, Ph. D. Med., leading Researcher, Head of the Department of Radiology SaintPetersburg State Research Institute of Phthisiopulmonology.

Address: 2-4, Ligovsky pr., St. Petersburg, 191036, Russia.

Phone number: +7 (812) 775-75-55. E-mail: spbniifrentgen@mail.ru

ORCID.org/0000-0003-3251-4084

\section{Финансирование исследования и конфликт интересов.}

Исследование не финансировалось какими-либо источниками. Автор заявляет, что данная работа, ее тема, предмет и содержание не затрагивают конкурирующих интересов.

Дата поступления статьи в редакцию издания: 23.06.2021 г.

Дата одобрения после рецензирования: 01.07.2021 г.

Дата принятия статьи к публикации: 01.07.2021 г. 